\title{
Ketamine Pharmacokinetics and Pharmacodynamics Are Altered by P-Glycoprotein and Breast Cancer Resistance Protein Efflux Transporters in Mice ${ }^{\S}$
}

\author{
Samit Ganguly, John C. Panetta, Jessica K. Roberts, and Erin G. Schuetz \\ Department of Pharmaceutical Sciences, St. Jude Children's Research Hospital, Memphis, Tennessee, (S.G., J.C.P., J.K.R., E.G.S.); \\ and Cancer and Developmental Biology Track, University of Tennessee Health Science Center, Memphis, Tennessee (S.G.)
}

Received August 30, 2017; accepted April 16, 2018

\begin{abstract}
To understand the systemic impact of breast cancer resistance protein (Bcrp) and P-glycoprotein (Pgp) deletion, untargeted metabolomics was performed on cerebral spinal fluid (CSF) and plasma of wild-type (WT) and Pgp and Bcrp double-knockout (dKO) rats anesthetized with ketamine-xylazine. We unexpectedly found elevated ketamine levels in both CSF and plasma of dKO versus WT rats. Therefore, the effect of these transporters was investigated on the 1) oral and intraperitoneal serum pharmacokinetics (PK) of ketamine, using a liquid chromatography method (high-performance liquid chromatography with ultraviolet detection), and 2) the anesthetic effect of ketamine using a duration of loss-of-righting reflex (dLORR) test in WT, Bcrp knockout (KO), Pgp KO, and Pgp/Bcrp dKO mice. The
\end{abstract}

\section{Introduction}

Ketamine is a clinically useful anesthetic that is used either alone or in combination with other drugs in different surgeries (Sleigh et al., 2014). Ketamine is also useful for the management of neuropathic and postoperative pain (Niesters et al., 2014; Barrett and Schriger, 2016). Recent literature reports have identified a long-lasting antidepressant activity of ketamine at subanesthetic doses (Machado-Vieira et al., 2009). In addition to its clinical use, ketamine is also a recreationally abused drug and chronic ketamine use causes kidney pathology, ulcerative cystitis (Shahani et al., 2007), and bladder toxicity (Chu et al., 2008).

Pharmacologically, ketamine is an $N$-methyl-D-aspartate receptor antagonist that binds to the phencyclidine binding pocket of the receptor (Mion and Villevieille, 2013) to elicit anesthesia. However, ketamine is also known to interact with other pharmacological targets (Sleigh et al., 2014). For example, ketamine was shown to have rapid-onset antidepressant activity after metabolism to $(2 R, 6 R)$-hydroxynorketamine, which activates $\alpha$-amino-3-hydroxy-5-methyl-4-isoxazole propionic acid receptors (Zanos et al., 2016).

This work was supported in part by the National Institutes of Health National Cancer Institute [Cancer Center Support Grant P30-CA21765] and the American Lebanese Syrian Associated Charities.

https://doi.org/10.1124/dmd.117.078360.

S This article has supplemental material available at dmd.aspetjournals.org.
PK data demonstrated a significantly increased oral bioavailability and serum exposure of ketamine in dKO > Pgp KO > Bcrp KO mice compared with WT mice. Intraperitoneal ketamine-induced dLORR was significantly longer in dKO > Pgp KO > Bcrp KO > WT mice compared with WT mice. Inhibition of Bcrp and Pgp in WT mice using the dual Pgp/Bcrp inhibitor elacridar increased the ketamine-induced dLORR compared with vehicle-treated mice. The ketamine intracellular concentration was significantly decreased in Madin-Darby canine kidney II BCRP/PGP cells compared with the parental cells. In total, these results demonstrate that ketamine appears to be a dual Pgp/Bcrp substrate whose PK and pharmacodynamics are affected by Pgp and Bcrp-mediated efflux.

Ketamine pharmacokinetics (PK) in humans are well described by a two- or three-compartment PK model (Fanta et al., 2015; Sherwin et al., 2015 ) with a high volume of distribution, high clearance, and low plasma protein binding (Mion and Villevieille, 2013). Ketamine is metabolized primarily by CYP2B6 and CYP3A4 to form norketamine, which is further metabolized to hydroxynorketamine and its glucuronide (Hijazi and Boulieu, 2002). Ketamine has a low oral bioavailability that can be partially explained by its high metabolism observed with the oral route compared with intravenous or intramuscular routes (Fanta et al., 2015). In addition, modifications in ketamine metabolism have been reported to cause potential drug-drug interactions (Peltoniemi et al., 2012).

Breast cancer resistance protein (Bcrp/Abcg2) and P-glycoprotein (Pgp/Abcb1a) are membrane-bound efflux transporters that are present in a wide variety of tissues such as the blood-brain barrier and bloodcerebrospinal fluid (CSF) barrier in the brain, intestinal epithelial cells, and the bile canalicular membrane and kidney tubules. These transporters recognize a wide variety of chemicals as their substrates and reduce substrate systemic exposure by decreasing absorption (intestine) and increasing elimination (liver and kidney). Pgp and BCRP expression at the blood-brain barrier and blood-CSF barrier decreases substrate movement from the blood to the brain and CSF, respectively. Thus, inhibition of Bcrp and/or Pgp can lead to systemic overexposure of their substrates and cause toxicity. A wide variety of drugs as well as chemicals from food or drinks are known to inhibit these transporters and can therefore lead to potential drug-drug or drug-food interactions (Varma

ABBREVIATIONS: $\mathrm{AUC}_{\text {inf }}$, area under the time-concentration curve extrapolated to infinity; Bcrp, breast cancer resistance protein; CSF, cerebral spinal fluid; dKO, double knockout; dLORR, duration of loss-of-righting reflex; FVB, Friend virus B; HPLC, high-performance liquid chromatography; HPMC, hydroxypropylmethylcellulose; IS, internal standard; KO, knockout; LC, liquid chromatography; LORR, loss-of-righting reflex; MDCKII, Madin-Darby canine kidney II; MS/MS, tandem mass spectrometry; PD, pharmacodynamics; Pgp, P-glycoprotein; PK, pharmacokinetics; WT, wild type. 
et al., 2003; Kusuhara et al., 2012). Moreover, the BCRP Q141K inactivating allele is common in some populations, with as many as $6 \%$ of Asians homozygous for this loss-of-function allele (Zamber et al., 2003).

Because of the large number of Bcrp and Pgp substrates and inhibitors as well as the potential for some chemicals to be dual substrates for both transporters, screening drugs in development for Bcrp and Pgp transport and inhibition capability is one of the U.S. Food and Drug Administrationrequired drug approval criteria (Tweedie et al., 2013). Some in vivo studies in rodents as well as in humans have suggested a potential interaction of ketamine with Bcrp and/or Pgp (Sato et al., 2007; Lilius et al., 2015). Given the clinical and recreational use of ketamine and the significant interest in ketamine as an effective antidepressant, we sought to identify whether ketamine is a substrate of Bcrp and/or Pgp. Using mice with and without these two transporters, we report for the first time that ketamine is an apparent dual Pgp/Bcrp substrate whose PK and pharmacodynamic (PD) properties are affected by both Pgp and Bcrp.

\section{Materials and Methods}

\section{Drugs and Chemicals}

Ketamine hydrochloride injection USP $(100 \mathrm{mg} / \mathrm{ml})$ was purchased from JHP Pharmaceuticals (Rochester, MI). Elacridar was purchased from Astatech Inc. (Bristol, PA). Hydroxypropylmethylcellulose (HPMC) and Tween 80 were purchased from Sigma-Aldrich (St Louis, MO). Promega Reporter Lysis Buffer $(5 \times)$, Pierce BCA protein assay reagent (A and B), bupropion, acetonitrile, formic acid, and ammonium hydroxide were purchased from Fisher Scientific (Pittsburgh, PA). ${ }^{3} \mathrm{H}-$ ketamine was obtained from American Radiolabeled Chemicals (St. Louis, MO).

\section{Animals and Cell Lines}

Wild-type (WT) and Bcrp/Pgp (Abcg2-Abcb1a) double-knockout (dKO) male Sprague-Dawley rats, weighing 200-250 g, were obtained from SAGE Laboratories (Horizon Discovery, St. Louis, MO). Male Bcrp (Abcg2) knockout (KO), Pgp (Abcb1a/b) KO, Pgp/Bcrp (Abcb1a/1b/Abcg2) dKO, and WT Friend virus $\mathrm{B}$ (FVB) mice were obtained from Taconic farms (Germantown, NY). Rats used in the experiments were 12 weeks old and mice were 10-16 weeks old. All animals were provided water and food ad libitum. Ketamine hydrochloride stock solution $(100 \mathrm{mg} / \mathrm{ml})$ was diluted with filtered autoclaved water to the appropriate dilution for dosing. Elacridar $(10 \mathrm{mg} / \mathrm{ml})$ was formulated by suspending elacridar in a solution of $0.5 \%$ HPMC with $1 \%$ Tween 80 . A 1.5 -inch 20 -gauge feeding needle (Fisher Scientific) was used to perform oral dosing. The Institutional Animal Care and Use Committee of St. Jude Children's Research Hospital in accordance with the U.S. National Institutes of Health guidelines approved all experimental procedures.

Madin-Darby canine kidney II (MDCKII) parental cells and derivative cells stably overexpressing both human PGP and BCRP were kindly provided by Dr. Alfred H. Schinkel's laboratory (The Netherlands Cancer Institute, Amsterdam, Netherlands) and were cultured as described (Poller et al., 2011).

\section{Drug Formulations for Animal Experiments}

Ketamine hydrochloride stock solution $(100 \mathrm{mg} / \mathrm{ml})$ was diluted with filtered autoclaved water to the appropriate dilution for oral or intraperitoneal dosing. Elacridar (solution) formulation was prepared by suspending the appropriate amount of elacridar in $0.5 \%$ HPMC with $1 \%$ Tween 80 solution to achieve a final concentration of $10 \mathrm{mg} / \mathrm{ml}$.

\section{Estimation of Ketamine Exposure in Rat CSF and Plasma}

Male WT and Pgp/Bcrp dKO rats ( $n=8$ per group) were anesthetized with intraperitoneal ketamine $(100 \mathrm{mg} / \mathrm{kg})$ and xylazine $(10 \mathrm{mg} / \mathrm{kg})$ formulated in water, at a dose volume of $10 \mathrm{ml} / \mathrm{kg}$ body weight. CSF was collected from the cisterna magna immediately after the anesthesia was achieved (approximately 7 minutes), followed by blood collection by cardiac puncture. Blood samples were centrifuged and plasma was separated from the blood. Both plasma and CSF samples were immediately frozen on dry ice, stored at $-80^{\circ} \mathrm{C}$, and shipped to Metabolon (Research Triangle Park, NC) for metabolomic analysis. Liquid chromatography (LC)-tandem mass spectrometry (MS/MS) sample analysis, data normalization, and analysis were performed at Metabolon as described in other reports (Evans et al., 2009). Normalized ketamine peak area intensity values were compared between the WT and dKO rats in both CSF and plasma.

\section{PK Study of Ketamine in WT, Bcrp KO, Pgp KO, and dKO Mice after Oral and Intraperitoneal Dosing at $100 \mathrm{mg} / \mathrm{kg}$}

A serum PK study was conducted after ketamine intraperitoneal and oral dosing of WT, Bcrp KO, Pgp KO, and dKO mice at a $100 \mathrm{mg} / \mathrm{kg}$ dose of ketamine. For the intraperitoneal PK study, six mice from each genotype were divided into two groups of three mice each. After the animals were dosed intraperitoneally, blood was collected from one group of mice at 2,10, and 40 minutes and from another group of mice at 5,20 , and 60 minutes. At each time point, $50 \mu 1$ blood was withdrawn from each mouse from the saphenous vein into microvette capillary blood collection tubes (Sarstedt, obtained from Fisher Scientific). Samples were kept at room temperature and were centrifuged at 10,000 rpm for 5 minutes to obtain serum, which was immediately separated and stored at $-80^{\circ} \mathrm{C}$ until further analysis. For the oral PK study, six mice from each genotype were separated into two groups (three mice in each group). After oral dosing, blood samples were collected at alternative time points from each group similar to the intraperitoneal study. After a 2-week washout period, the same oral study was repeated by altering the groups and time points such that all time points were collected from each mouse. The two oral PK data sets were first analyzed separately and compared to identify any significant interday variation; after we confirmed that there was no interday difference in the PK profile, the data from the two collection dates were merged to obtain complete PK profiles for each mouse.

\section{Determination of Ketamine Concentration in Mouse Serum with High- Performance Liquid Chromatography with Ultraviolet Detection}

Serum samples from the intraperitoneal ketamine PK studies in mice were treated with chilled acetonitrile (1:5); samples were vortexed and centrifuged for 15 minutes at $4000 \mathrm{rpm}$ to precipitate the protein and extract ketamine. After centrifugation, the supernatant was collected and further centrifuged for sample cleaning. The resultant final supernatant was then diluted 1:1 with water/acetonitrile and a $10 \mu \mathrm{l}$ sample was injected onto the high-performance liquid chromatography (HPLC) system for further analysis. For oral PK, a 1:2.5 initial dilution with chilled acetonitrile was used, followed by centrifugation and protein precipitation and recentrifugation of the supernatant for sample cleaning. The final supernatant was injected onto the HPLC system (Shimadzu Prominence, Kyoto, Japan) consisting of an LC-20AD binary high-pressure gradient pump, an SIL$20 \mathrm{AC}_{\mathrm{HT}}$ auto sampler, and a SPD-20AV UV detector. Bupropion $(100 \mu \mathrm{g} / \mathrm{ml})$ was added as an internal standard (IS) to all of the samples prior to extraction and was extracted along with ketamine. Chromatographic separation of ketamine along with the IS was achieved using a Hypersil ODS C18 column $(150 \times 4 \mathrm{~mm} ; 5 \mu \mathrm{m}$ particle size) fitted with a Hypersil ODS (C18) Javelin Guard Column $(10 \times 4 \mathrm{~mm}$; $5 \mu \mathrm{m}$ particle size) and a gradient elution method with a run time of 10 minutes. The mobile phase for elution was 60:40 ammonium formate buffer $(20 \mathrm{mM}) /$ acetonitrile (Moore et al., 2001) and the following gradient was used: $0-3$ minutes, $40 \%$ acetonitrile; $3-8$ minutes, $60 \%$ acetonitrile; 8 to 9 minutes, $40 \%$ acetonitrile; and the run stopped at 10 minutes. Retention times for ketamine and bupropion were 5.2 and 7.1 minutes, respectively. The ketamine peak was identified and absorbance was measured at $210 \mathrm{~nm}$ (Bolze and Boulieu, 1998). The analyte/IS height ratio was used for preparation of calibration and measurement of unknown concentrations. Extraction efficiency was $>90 \%$ throughout the analysis. The calibration ranges for intraperitoneal PK and oral PK analysis were 0.39-200 and $0.19-100 \mu \mathrm{g} / \mathrm{ml}$, respectively.

\section{Measurement of the Ketamine Brain/Serum Ratio after Intraperitoneal Ketamine Dosing}

Male FVB WT, Pgp KO, and Pgp/Bcrp dKO mice were dosed with intraperitoneal ketamine $(100 \mathrm{mg} / \mathrm{kg})$ plus $1 \mu \mathrm{Ci}{ }^{3} \mathrm{H}$-ketamine tracer 1 hour before and again 15 minutes before sacrifice. Mice were euthanized by $\mathrm{CO}_{2}$ inhalation, blood was collected and serum separated, and brain tissue was weighed and mixed with an equivalent volume of phosphate-buffered saline and homogenized. Brain homogenate and serum were mixed with scintillation fluid and total radioactivity was determined by liquid scintillation counting. The ${ }^{3} \mathrm{H}$-ketamine brain/serum ratio is in disintegrations per minute per gram brain tissue homogenate divided by disintegrations per minute per milliliter of serum. 
In another similar study, FVB male mice (WT, Pgp KO, and Pgp/Bcrp dKO) were sacrificed 15 minutes after they were dosed with $100 \mathrm{mg} / \mathrm{kg}$ ketamine intraperitoneally. Brain and blood were collected from each mouse after sacrifice. Brain tissues were weighed and were homogenized with $20 \mathrm{mM}$ ammonium formate buffer, and $9 \mu \mathrm{l}$ brain tissue homogenate plus $1 \mu \mathrm{l}$ IS was extracted using chilled acetonitrile. The supernate from the extracted samples was mixed with an equal volume of acetonitrile/water (1:1) and injected onto the HPLC-UV system for measurement of ketamine against standards prepared similarly from untreated mouse brain homogenate. The serum was separated from blood samples and the ketamine concentration was measured by an HPLC-UV-based method as described previously. The brain/serum ratio was obtained by dividing the weight-normalized brain tissue concentration by the serum concentration of ketamine.

\section{PK Data Analysis and Interpretation}

Noncompartmental Analysis. Initial analysis of both intraperitoneal and oral PK study data was performed by a noncompartmental approach using the PK package in R statistical software (R Project for Statistical Computing, Vienna, Austria) (Jaki and Wolfsegger, 2011) to determine whether there were significant differences in ketamine serum exposure between WT and Bcrp KO, Pgp KO, and dKO mice. A serial sacrifice design was assumed to compute the area under the time-concentration curve extrapolated to infinity $\left(\mathrm{AUC}_{\mathrm{inf}}\right)$ and the associated variability as described (Jaki and Wolfsegger, 2011).

Nonlinear Mixed Effect Modeling. Upon identification of a significant exposure difference between genotypes in the oral PK study by noncompartmental analysis, the oral and intraperitoneal PK data were combined and analyzed with nonlinear mixed-effects modeling implemented in Monolix 4.3.3 software (Lixoft, Antony, France) using a stochastic approximation expectation maximization algorithm (Kuhn and Lavielle, 2005) combined with a Markov chain Monte Carlo procedure. Population analysis of our PK data did not show any difference in ketamine serum PK between WT and Bcrp KO mice; thus, these two genotypes were combined in the final model. Of 144 data points in the oral PK study, four concentrations were below the lowest calibration standard concentration $(0.19 \mu \mathrm{g} / \mathrm{ml})$ and these were removed from the analysis. One- and two-compartment models were evaluated. The goodness-of-fit-criteria and minimization of the Akaike information criterion were used to select the base model. The effect of overparameterization was also assessed using the condition number and the Bayesian information criterion. Covariate analysis included the effect of genotype as a categorical covariate following an exponential model. The effect of genotype on individual parameters was determined by a forward addition process with a decrease in Objective Function Value $\geq 3.84$ considered significant at $P=0.05$ for one degree of freedom, based on the $\chi^{2}$ distribution. Variability in observed data from the model-predicted population mean data was considered to be due to between-subject variability and residual unexplained variability. The following equation was used to describe betweensubject variability (eq. 1):

$$
P_{i}=\theta_{\text {pop }} \times \exp \left(\eta_{\text {pop }}\right)
$$

where $P_{i}$ is the PK parameter of the $i^{\text {th }}$ individual, $\theta_{\mathrm{pop}}$ is the population mean for $P$, and $\eta$ is the normally distributed between-subject random effect with a mean of zero and variance of $\omega^{2}$. Constant, proportional, and combined constant and proportional error models were tested for residual unexplained variability, and the final model used the proportional error model that follows eq. 2 :

$$
Y_{i j}=\hat{Y}_{i j}+Y_{i j}^{*} \varepsilon
$$

where $Y_{i j}$ is the observed concentration of $i^{\text {th }}$ individual at time $j, \hat{Y}_{i j}$ is the individual predicted concentration, and $\varepsilon$ is the proportional error that is normally distributed with a mean of zero and variance of $\sigma^{2}$.

Diagnostic plots were used for the final assessment of the model. Correlations between the observed and model-predicted concentrations were evaluated and used as screening criteria for model selection. Population-weighted residuals or individual-weighted residuals were plotted against time and the population or individual predicted ketamine concentration. The normalized prediction distribution error plotted against time and the population-predicted ketamine concentration was also evaluated for model misspecification assessment. Apart from the diagnostic plots, the final model selection also included a comparison of objective function values and variability associated with parameter estimates.
Comparison of Ketamine-Induced Duration of Loss-of-Righting Reflex Between WT, Bcrp KO, Pgp KO, and dKO Mice

The ketamine-induced duration of loss-of-righting reflex (dLORR) was studied as described (Sato et al., 2004). Briefly, animals were administered ketamine $(50,100$, or $200 \mathrm{mg} / \mathrm{kg}$ ) intraperitoneally or by oral gavage. After 4 minutes of waiting time (to reduce manual stimulation), the mice were placed on their back. The loss-of-righting reflex (LORR) was defined to have taken place when the mouse could not right itself for at least 20 seconds. The dLORR was then measured until the mouse woke up spontaneously and the dLORR was compared between WT, Bcrp KO, Pgp KO, and dKO mice. In a separate study, WT mice were orally administered elacridar $(100 \mathrm{mg} / \mathrm{kg})$ or vehicle 1.5 hours prior to intraperitoneal administration of $50 \mathrm{or} 100 \mathrm{mg} / \mathrm{kg}$ ketamine and ALORR was measured as described above.

\section{Intracellular Uptake of Radiolabeled Ketamine in MDCKII Cells Overexpressing Human PGP and BCRP}

MDCKII cells with or without BCRP and PGP were plated at $0.2 \times 10^{6}$ cells/well in a 24-well plate. The next day, the medium was removed and the cells were incubated with ${ }^{3} \mathrm{H}$-ketamine at $37^{\circ} \mathrm{C}$. At the selected time points, the cells were washed with chilled phosphate-buffered saline and incubated for 1 hour with lysis buffer, the cell lysates were collected, and the intracellular concentration of ${ }^{3} \mathrm{H}$-ketamine and protein concentration was determined in the lysate by the BCA protein assay. Results are expressed as picomoles of ketamine per milligram of protein lysate. First, we determined the time to reach an equilibrium of intracellular uptake by incubating both WT and transfected cells with $0.5 \mu \mathrm{M}^{3} \mathrm{H}$ ketamine for 1 hour and measuring the intracellular radioactivity at 2, 5, 15,30 and 45 minutes. In a separate assay, the concentration-dependent uptake of ${ }^{3} \mathrm{H}$ ketamine up to the time of equilibrium was determined in MDCKII and BCRPPGP-MDCKII cells by incubating the cells with different concentrations of ${ }^{3} \mathrm{H}$-ketamine until they reached equilibrium and then measuring the intracellular concentration of ${ }^{3} \mathrm{H}$-ketamine. The intracellular ketamine concentration was calculated and compared between the MDCKII and BCRP-PGP-MDCKII cells.

\section{Statistical Analyses}

GraphPad QuickCalcs (GraphPad Software Inc., La Jolla, CA) was used to perform the Grubbs' outlier test to remove outlier data from the analysis. Statistical analyses of significance for CSF and plasma ketamine exposure data in rats, all PD (dLORR) data, in vitro cell uptake study data, and noncompartmental analysis were performed in GraphPad Prism software (version 5.02). The CSF and plasma peak intensity of ketamine in $\mathrm{dKO}$ rats and WT rats was compared using the unpaired $t$ test with the Welch correction. All dLORR study data were analyzed using the Mann-Whitney $U$ test. Intracellular uptake study results were analyzed using an unpaired $t$ test. The noncompartment-determined $\mathrm{AUC}_{\mathrm{inf}}$ was analyzed using a one-way analysis of variance with the Newman-Keuls multiple-comparisons test. Significance for all analyses was equal to $P<0.05$.

\section{Results}

\section{Deletion of Both Bcrp and Pgp Results in Greater Ketamine} Exposure in the CSF and Plasma of dKO Rats

We performed a metabolomic comparison analysis of the CSF and plasma from WT and Bcrp/Pgp dKO rats anesthetized with a cocktail of ketamine-xylazine. A full report of the metabolomics results will be published elsewhere. Ketamine levels (measured by LC-MS/MS) were significantly higher in the CSF (1.74 times) and plasma (1.93 times) of dKO compared with WT rats (Fig. 1). These results suggested that ketamine might be a substrate of Bcrp and/or Pgp. The metabolomic analysis did not identify the presence of xylazine in either CSF or plasma from either WT or Pgp/Bcrp dKO rats.

\section{Ketamine Oral Serum Exposure Is Higher in Mice Lacking Bcrp and Pgp}

To determine whether Bcrp and Pgp can affect the PK of ketamine, we collected serum after $100 \mathrm{mg} / \mathrm{kg}$ intraperitoneal dosing to WT, Bcrp KO, Pgp KO, and dKO mice. Serum concentrations of ketamine at the 


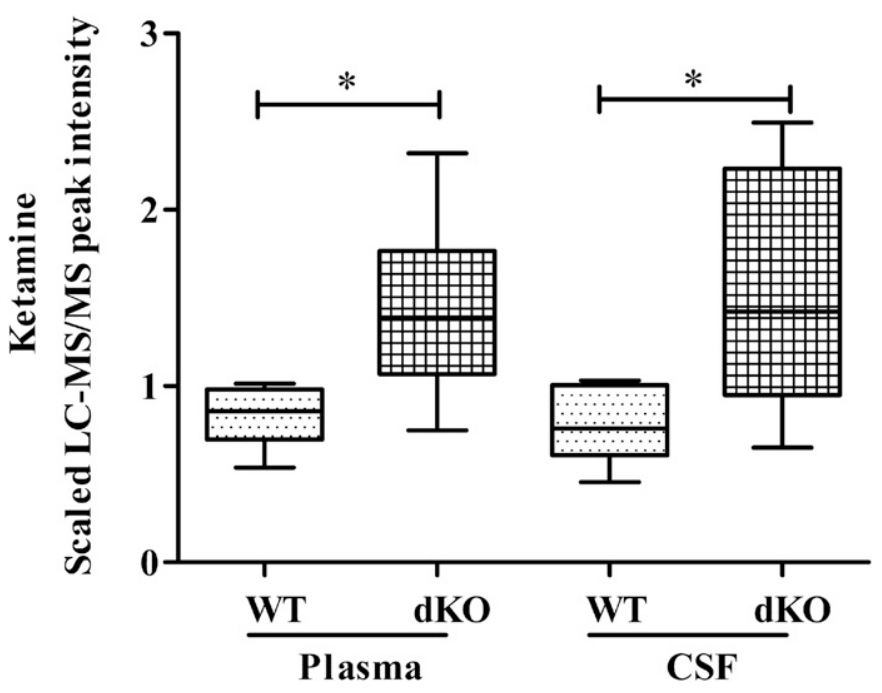

Fig. 1. Elevated ketamine CSF and plasma levels in Pgp/Bcrp dKO versus WT rats. Ketamine levels in male Sprague-Dawley rats after intraperitoneal administration of $100 \mathrm{mg} / \mathrm{kg}$ ketamine. Data are plotted as the median LC-MS/MS peak intensity \pm range $(n=8 /$ genotype). The plasma and CSF median peak intensity in dKO rats was compared with the corresponding median peak intensity from WT rats using the unpaired $t$ test with the Welch correction. ${ }^{*} P<0.05$.

selected time points were similar between all four genotypes (Fig. 2A). Ketamine serum exposure, as measured by the area under the timeconcentration curve $\left(\mathrm{AUC}_{\mathrm{inf}}\right)$ by a noncompartmental analysis, was also similar between all of the genotypes (Fig. 2B).
Since Bcrp and Pgp have a higher impact on their substrates' serum/plasma exposure after oral compared with intraperitoneal dosing (Lagas et al., 2009), we collected serum after a $100 \mathrm{mg} / \mathrm{kg}$ dose of ketamine was administered orally to WT, Bcrp KO, Pgp KO, and $\mathrm{dKO}$ mice. Ketamine serum concentrations were significantly higher in dKO mice compared with the WT mice (Fig. 2C). Ketamine serum exposures $\left(\mathrm{AUC}_{\mathrm{inf}}\right)$, calculated by the noncompartmental analysis method, were significantly higher in dKO (1.96-fold) and Pgp KO (1.52-fold) mice and were slightly higher in Bcrp KO mice (1.20-fold) compared with WT mice (Fig. 2D). The ketamine serum concentration at the 60 -minute time point could be detected in only two of six WT mice but was detected in all of the Bcrp KO, Pgp KO, and $\mathrm{dKO}$ mice. Thus, the last time point for the observed concentration is different between WT and other $\mathrm{KO}$ mice. Hence, the $\mathrm{AUC}_{\mathrm{inf}}$ was used to compare the exposure between all genotypes. Both Pgp $\mathrm{KO}$ as well as Bcrp KO mice showed higher concentrations of ketamine than WT mice at earlier time points, indicating a possible alteration in absorption and possibly driving the observed difference in $\mathrm{AUC}_{\text {inf }}$ with the WT mice. Detailed noncompartmental analysis parameters for both intraperitoneal and oral PK studies are listed in Supplemental Table 1. Although the noncompartmental analysis allowed comparison of concentration-time curve properties, there was no assumption of the compartment and there was no information about which PK parameters could be causing the difference in $\mathrm{AUC}_{\text {inf. }}$. However, an alteration in bioavailability was suggested, because dividing the oral $\mathrm{AUC}_{\mathrm{inf}}$ by the intraperitoneal $\mathrm{AUC}_{\mathrm{inf}}$ gave an $(\mathrm{F})$ for the mice that was greatest in the $\mathrm{dKO}$ (approximately 27\%) > Pgp KO (approximately 24\%) > Bcrp KO (approximately 17\%) = WT (approximately 14\%).
A
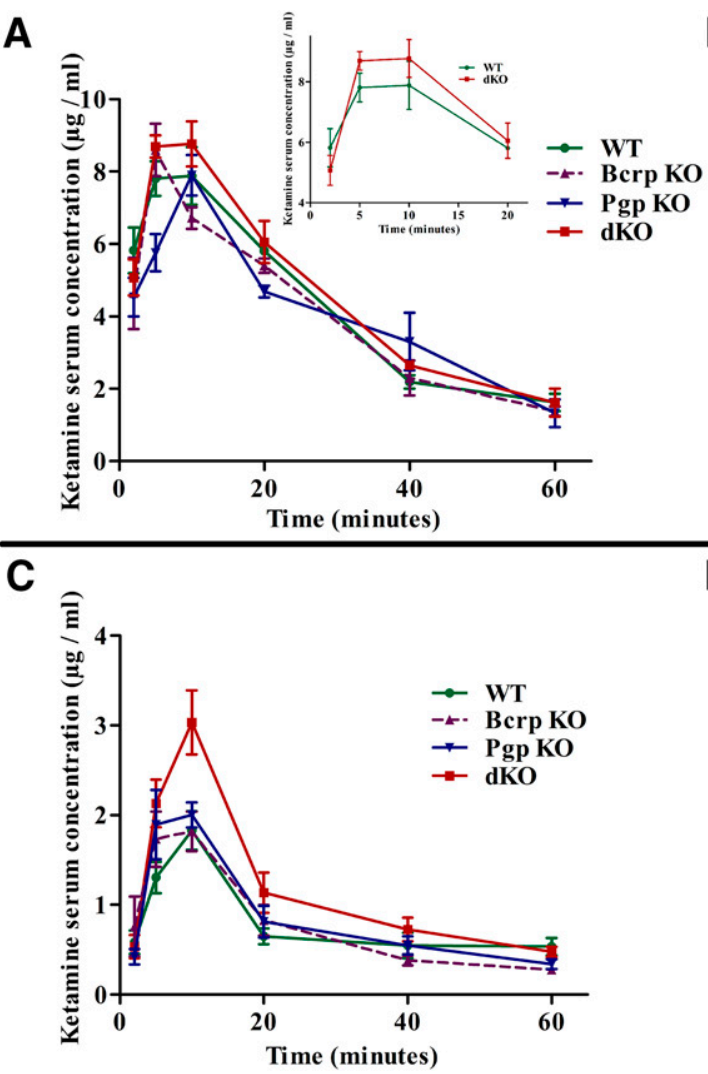

B

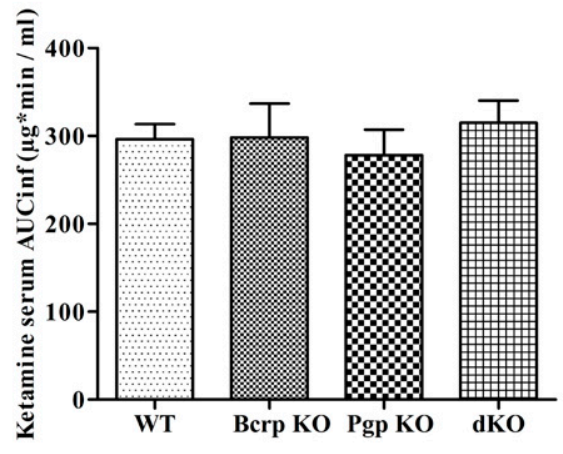

IP
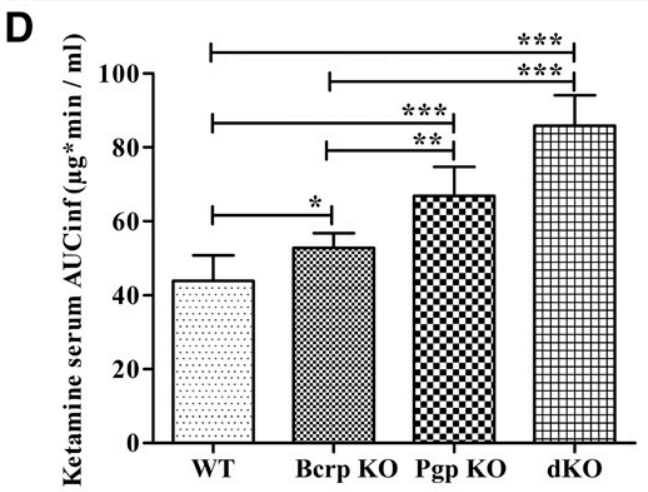

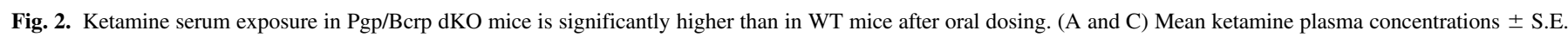

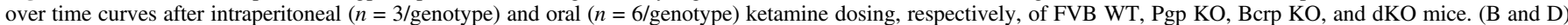

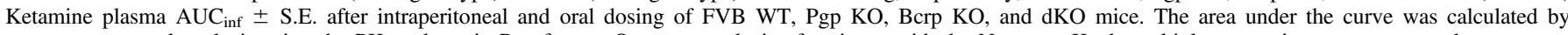

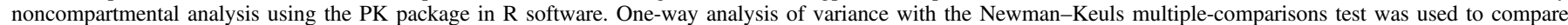
AUC $_{\text {inf. }}{ }^{*} P<0.05$; $* * P<0.01 ; * * * P<0.001$. IP, intraperitoneal; PO, oral. 
Population PK Analysis Demonstrates an Increase in Ketamine Oral Bioavailability in Pgp/Bcrp dKO and Pgp KO Mice Compared with WT and Bcrp KO Mice

To better understand the impact of Bcrp- and Pgp-mediated efflux on PK properties of ketamine, we combined both oral and intraperitoneal PK data in a simultaneous model using a population PK approach. This model also assumed that bioavailability after intraperitoneal dosing was equal to one. The final model was a one-compartment distribution with zero-order absorption for oral and first-order absorption for the intraperitoneal dosing and first-order elimination from the central compartment (Supplemental Fig. 1). The one-compartment model was sufficient to describe these data (Supplemental Figs. 2-4). The final parameters of population analysis are shown in Table 1. Transporter genotypes had no effect on clearance or volume of distribution. The small difference in ketamine PK between Bcrp KO versus WT mice observed by noncompartmental analysis was not observed with the population approach, likely due to variability in the result. However, both Pgp KO and $\mathrm{dKO}$ genotypes had a significant impact on population bioavailability $(P<0.001)$. Oral bioavailability $(\mathrm{F})$ for the WT population was $17.5 \%$, which significantly increased to $22 \%$ in Pgp KO mice and increased further to $29 \%$ in dKO mice. Hence, altered ketamine PK was observed after oral administration to mice lacking Pgp singly or in combination with Bcrp. This difference translates to a difference in the post hoc estimated area under the curve between these groups.

\section{Ketamine-Induced dLORR Was Significantly Increased in Mice Lacking Bcrp and Pgp}

The difference in oral PK between WT and Bcrp and/or Pgp KO mice, as well as the higher plasma and CSF abundance of ketamine in dKO compared with WT rats, led us to investigate whether ketamine PD is also affected by Bcrp and Pgp. We used the ketamine-induced LORR model in the mouse to study the effect of Bcrp and Pgp on ketamine PD. After intraperitoneal ketamine administration, the median dLORR in dKO mice was longer than in WT (2.6-fold), Bcrp KO (2.1-fold), and Pgp KO (1.3-fold) mice (Fig. 3A; Supplemental

TABLE 1

Population PK parameter estimates

\begin{tabular}{lccc}
\hline \multicolumn{1}{c}{ Parameter } & Mean Parameter Estimate & S.E & $P$ Value \\
\hline F_pop & 0.175 & 0.0072 & \\
$\theta$ _Pgp KO & 0.227 & 0.062 & 0.00028 \\
$\theta$ _KO & 0.514 & 0.062 & $<1 \mathrm{e}-10$ \\
T $k$ 0_pop (1/h) & 0.104 & 0.0092 & \\
F2_pop & 1 fixed & N.E. & \\
$k_{\text {a_pop }(1 / \mathrm{h})}$ & 19.1 & 1.6 & \\
V1_pop $(1 / \mathrm{kg})$ & 9.32 & 0.35 & \\
CL_pop $(1 / \mathrm{h}$ per kilogram) & 20.2 & 0.72 & \\
$\omega \_$BSV & & & \\
F & 0.0276 & 0.077 & \\
T $k 0$ & 0.32 & 0.077 & \\
F2 & N.E. & N.E. & \\
$k_{\text {a }}$ & 0.091 & 0.14 & \\
V1 & 0.0857 & 0.061 & \\
CL & 0.0781 & 0.056 & \\
$\sigma$-RUV & & & \\
Proportional & 0.341 & 0.022 & \\
F_(WT and Bcrp KO) & 0.175 & 0.0072 & \\
F_(Pgp KO) & 0.22 & 0.011 & \\
F_(dKO) & 0.293 & 0.015 & \\
& & &
\end{tabular}

CL_pop, population mean clearance; F, oral bioavailability; F_pop, population mean oral bioavailability; F2_pop, population mean intraperitoneal bioavailability (which was assumed to

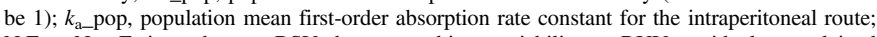
N.E. : Not Estimated., ; $\omega$ BSV, between-subject variability; $\sigma$-RUV, residual unexplained variability; $\theta \_$Pgp $\mathrm{KO}$ and $\theta_{-} \mathrm{dKO}$, estimated coefficient of the effect of categorical covariate Pgp $\mathrm{KO}$ and $\mathrm{dKO}$ on the oral bioavailability; T $k 0$ _pop, population mean oral zero-order absorption rate constant; V1_pop, population mean volume of distribution.
Table 2). In addition, the median dLORR was 2-fold longer in Pgp KO compared with WT mice. These results indicate that both Pgp and Bcrp influence ketamine PD as measured by ketamine-induced dLORR.

To determine whether ketamine brain concentrations were different in the transporter KO versus WT mice, we first administered ketamine intraperitoneally and measured brain and serum concentrations at 15 minutes after dosing ( $n=3$ in each genotype group). Our HPLC-UV analysis shows a median brain/serum ratio of 1.32 in all genotypes and there was no significant difference in brain/serum ketamine concentrations between genotypes (Supplemental Fig. 5). To understand whether the total concentration of ketamine and its metabolites is higher in KO mice compared with WT mice, we performed a whole brain homogenate ${ }^{3} \mathrm{H}$-ketamine radioactivity study. We administered the standard anesthetic dose of ketamine $(100 \mathrm{mg} / \mathrm{kg})$ intraperitoneally spiked with ${ }^{3} \mathrm{H}$-ketamine and measured the ${ }^{3} \mathrm{H}$-ketamine brain/serum ratio at 1.25 hours. The brain/serum ${ }^{3} \mathrm{H}$-ketamine radioactivity ratio was not significantly different between genotypes, with a median ratio of 1.45 in all mice. This result is similar to what we observed with the HPLC-UV analysis. Although there was a measurable increase in the level of ${ }^{3} \mathrm{H}$-ketamine in the brains of Pgp and Pgp/Bcrp dKO mice, the difference between genotypes did not reach statistical significance (Supplemental Fig. 5 ), despite the remarkable impact of the transporters on the PD effect of ketamine (Fig. 3A), and the significant increase in CSF ketamine (measured by LC-MS/MS) in Pgp/Bcrp dKO rats (Fig. 1). Although the difference in $\mathrm{Pgp} \mathrm{KO}$ versus dKO brain ${ }^{3} \mathrm{H}$-ketamine radioactivity reached statistical significance, the median dKO/Pgp $\mathrm{KO}$ radioactivity ratio was 0.85 , which could be due to differences in metabolite concentration between these two genotypes or even variability associated with analysis, dosing, or other reasons.

\section{Pharmacological Inhibition of Bcrp and Pgp by Elacridar Increased Intraperitoneal Ketamine-Induced dLORR in WT Mice}

Since we observed altered PK and PD of ketamine in mice with genetic deletion of Bcrp and/or Pgp, we investigated whether ketamine PD was also altered by pharmacological inhibition of these efflux transporters by using elacridar as a dual inhibitor of Bcrp and Pgp. Elacridar dosing and time schedules for pretreatment were based on previous reports that clearly demonstrated loss of Bcrp and Pgp function at the dose selected (Durmus et al., 2012; Sane et al., 2012; Hendrikx et al., 2014). WT mice were pretreated with vehicle or $100 \mathrm{mg} / \mathrm{kg}$ elacridar and ketamine was dosed intraperitoneally 1.5 hours later at 50 , 100 , and $200 \mathrm{mg} / \mathrm{kg}$ ( $n=6$ per dose group) and the duration of LORR was measured. The results of ketamine treatment alone in WT mice (Fig. 3B; Supplemental Table 3) demonstrated a dose-proportional increase in drug-induced dLORR from 50 to $100 \mathrm{mg} / \mathrm{kg}$, but there was a disproportionately longer increase in dLORR at $200 \mathrm{mg} / \mathrm{kg}$. Pretreatment with elacridar significantly increased dLORR in the WT mice treated with either 50 or $100 \mathrm{mg} / \mathrm{kg}$ ketamine. Based on the exceedingly long dLORR with $200 \mathrm{mg} / \mathrm{kg}$ ketamine alone, cotreatment at this dose with elacridar was not attempted to avoid toxicity. These results show that inhibition of Bcrp and Pgp with the dual inhibitor elacridar significantly increased ketamine-mediated dLORR in WT mice.

Ketamine-Induced dLORR Was Significantly Longer in dKO versus WT Mice after Oral Dosing of $200 \mathrm{mg} / \mathrm{kg}$ Ketamine

Because ketamine can be taken orally, we compared the effect of the drug transporters on its PD effect after oral administration. Oral ketamine dosing $(100 \mathrm{mg} / \mathrm{kg})$ was insufficient to cause a LORR in any of the mice (data not shown), likely because the ketamine serum concentration was lower after oral versus intraperitoneal dosing (4- and 7-fold lower in 

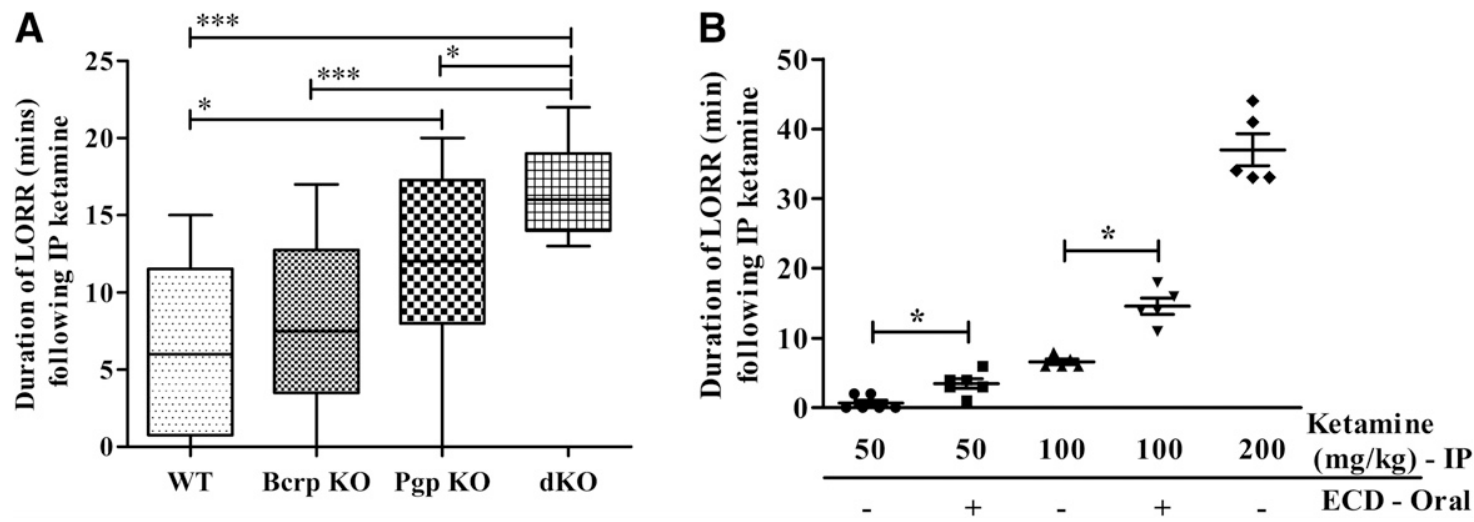

Fig. 3. The intraperitoneal ketamine-induced dLORR significantly increases in mice with absent or inhibited Pgp and Bcrp. (A) Comparison of ketamine-induced $(100 \mathrm{mg} / \mathrm{kg}$ body weight, i.p.) dLORR in WT, Bcrp KO, Pgp KO, and dKO FVB mice. (B) Ketamine-induced (50, 100 and $200 \mathrm{mg} / \mathrm{kg}$ body weight, i.p.) dLORR in WT mice with or without 1.5 -hour pretreatment with elacridar $(100 \mathrm{mg} / \mathrm{kg}$ body weight). Results are expressed as the median dLORR \pm range. Significance was calculated using the Mann-Whitney test. $* P<0.05 ; * * * P<0.001$. ECD, elacridar; IP, intraperitoneal

dKO and WT mice, respectively) (Supplemental Table 1). After oral ketamine treatment $(200 \mathrm{mg} / \mathrm{kg})$, WT mice were all awake within 4 minutes, whereas one of the dKO mice died and the others needed to be warmed to wake them after 30 minutes, preventing further analysis of this dose (data not shown).

\section{Ketamine Accumulation Is Lower in MDCKII Cells Transfected with Human BCRP and Pgp Compared with WT MDCKII Cells}

The time course of ${ }^{3} \mathrm{H}$-ketamine cellular uptake was first compared between MDCKII cells and MDCKII-PGP-BCRP cells stably expressing the human transporters (Poller et al., 2011). The ${ }^{3}$ H-ketamine cellular concentration was significantly greater in MDCKII parental cells compared with cells expressing BCRP and PGP at all time points, but uptake reached equilibrium by 20 minutes (Fig. 4A). Likewise, the ${ }^{3} \mathrm{H}$ ketamine intracellular concentration measured at 20 minutes was higher in the parental MDCKII cells compared with the dual BCRP/PGPexpressing cells between 0.1 and $5 \mu \mathrm{M}$ concentrations but not at the $10 \mu \mathrm{M}$ concentration (Fig. 4B). The data also indicated that the magnitude of difference in intracellular ${ }^{3} \mathrm{H}$-ketamine was greater at lower concentrations versus higher concentrations between the MDCKII cells with and without drug transporters. In support of these findings,
Keiser et al. (2018) recently published (while this manuscript was under review) in vitro studies showing that ketamine is a Pgp substrate.

\section{Discussion}

After the unexpected finding that the concentration of the anesthetic ketamine was elevated in the CSF and plasma of Pgp-Bcrp dKO compared with WT rats, this study was performed in mice to determine whether ketamine was a dual substrate for these transporters. Overall the results indicate that ketamine oral exposure increased in the following order: WT $<$ Bcrp KO $<$ Pgp KO $<$ dKO (Fig. 2). Likewise, the rank order for ketamine-induced dLORR was significantly longer in the $\mathrm{dKO}>\mathrm{Pgp} \mathrm{KO}>$ Bcrp KO > WT mice (Fig. 3). In summary, the absence of Pgp/Bcrp increased intraperitoneal ketamine-induced dLORR (Fig. 3), increased ketamine oral bioavailability (Fig. 2D; Table 1), and elacridar increased intraperitoneal ketamine-induced dLORR in WT mice (Fig. 3B), demonstrating that ketamine is an apparent substrate for both transporters.

There is mixed evidence in the literature that ketamine might interact with Pgp or Bcrp. Ketamine was previously screened for inhibitory activity against BCRP and PGP (Tournier et al., 2010) using MDCKII cells stably transfected with human BCRP or MDR1 (PGP) and was
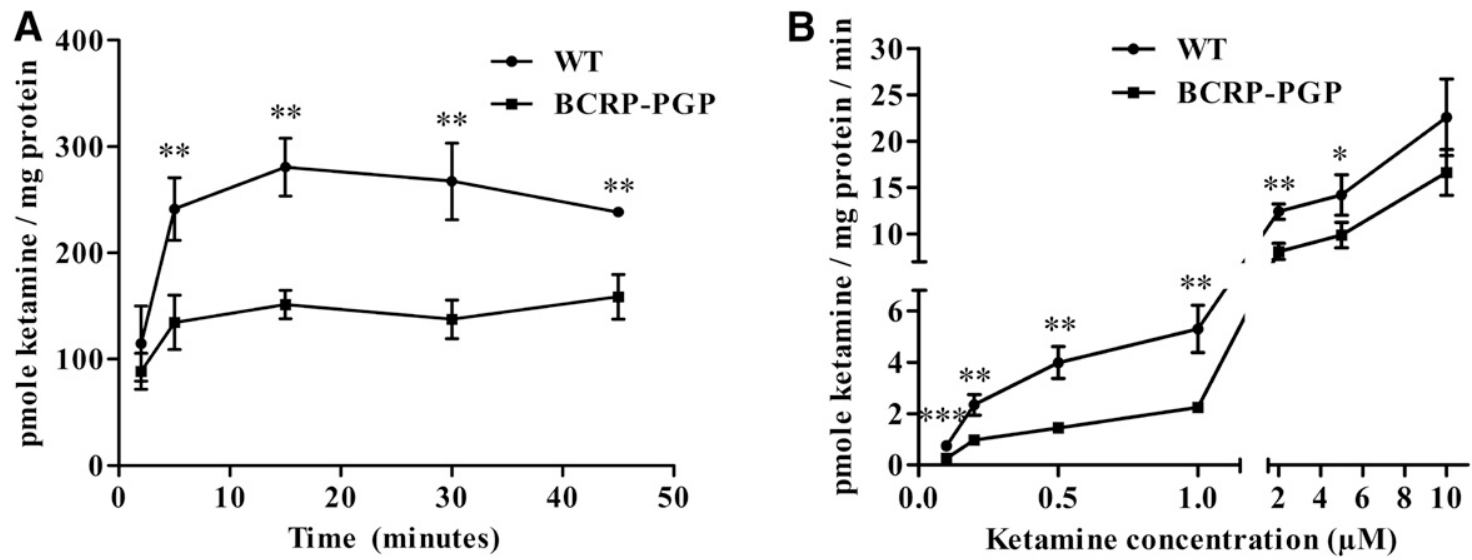

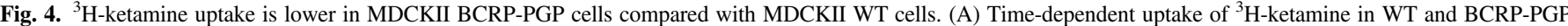

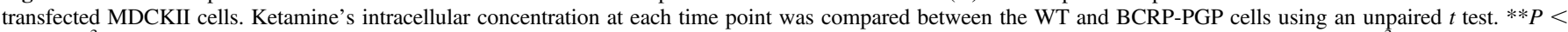

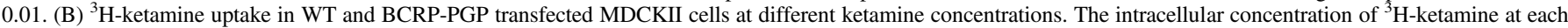
treatment was compared between the WT and BCRP-PGP cells using an unpaired $t$ test. $* P<0.05 ; * * P<0.01 ; * * * P<0.001$. 
found to not inhibit either transporter. However, high concentrations of cyclosporine increased the dLORR in mice (Sato et al., 2007), which could be due to cyclosporine's ability to inhibit ketamine-metabolizing cytochrome P450 enzymes as well as Pgp and Bcrp. The finding that female versus male rats show a longer ketamine-induced dLORR (Winters et al., 1986) might also reflect the fact that there is significant sexual dimorphism in rodent Bcrp expression, with males having significantly higher levels of Bcrp in the liver, intestine, and kidney compared with females (Merino et al., 2005). Coadministration of morphine (a Pgp substrate) and ketamine increased the brain concentration of ketamine (Lilius et al., 2015), which would be consistent with ketamine competing with morphine for Pgp transport.

We used dLORR to compare the ketamine-induced anesthetic/hypnotic effect in mice with different transporter genotypes, as dLORR can be used to assign dose-dependent PD effects (Sato et al., 2004). Although we observed a significantly increased intraperitoneal ketamine-induced dLORR in the dKO mice compared with WT, Bcrp $\mathrm{KO}$, and Pgp KO mice (Fig. 3A), we did not find a significant increase in serum PK after intraperitoneal ketamine dosing (Fig. 2A) in the transporter single $\mathrm{KO}$ and $\mathrm{dKO}$ mice. However, it is not unprecedented for some Pgp substrates to not show systemic PK differences between WT and Pgp KO mice after intraperitoneal dosing (e.g., dasatinib) (Lagas et al., 2009). Although ketamine levels were significantly higher in the plasma of Pgp/Bcrp $\mathrm{dKO}$ rats approximately 7 minutes after intraperitoneal dosing (Fig. 1), we were unable to detect a significant difference in ketamine PK after intraperitoneal dosing of WT or single transporter KO mice (Fig. 2A). This might have resulted from a number of factors, including interspecies variability in metabolism/transport and the difference in blood collection time after ketamine dosing between the two studies. PK parameters of ketamine in mice are not extensively reported in the literature. From the concentration-time data reported (Sato et al., 2004) after a $200 \mathrm{mg} / \mathrm{kg}$ intraperitoneal dose and (Zanos et al., 2016) after a $10 \mathrm{mg} / \mathrm{kg}$ intraperitoneal dose in mice, the plasma half-life of ketamine is approximately 25 minutes, which is similar to the results obtained in our study (Table 1 ). The $C_{\max }$ after intraperitoneal dosing of $10 \mathrm{mg} / \mathrm{kg}$ ketamine was reported to be between 0.55 and $1 \mu \mathrm{g} / \mathrm{ml}$ (Can et al., 2016; Zanos et al., 2016), whereas we observed a $C_{\max }$ of $7.88 \pm 1.38 \mu \mathrm{g} / \mathrm{ml}$ (Supplemental Table 1) after $100 \mathrm{mg} / \mathrm{kg}$ intraperitoneal dosing of ketamine, which is within the dose linear range of the reported data. Sato et al. (2004) reported the highest concentration of approximately $30 \mu \mathrm{g} / \mathrm{ml}$ at 10 minutes after $200 \mathrm{mg} / \mathrm{kg}$ intraperitoneal ketamine dosing in C57B1/6 mice (Sato et al., 2004), indicating a possible more than dose-proportional increase in ketamine exposure beyond the $100 \mathrm{mg} / \mathrm{kg}$ dose. Although ketamine bioavailability in humans after oral dosing has been reported to be between $7 \%$ and $20 \%$ (Peltoniemi et al., 2012; Fanta et al., 2015), bioavailability after oral dosing in mice is not well established. Assuming $100 \%$ bioavailability after intraperitoneal dosing, bioavailability after oral dosing in WT mice falls into the same range (F_Pop; Supplemental Table 1) in our study to that observed in humans.

We did not find a significant difference in the ketamine brain/plasma ratio between the mice with different transporter genotypes (Supplemental Fig. 5), and the increase in CSF concentrations in Pgp/Bcrp dKO versus WT rats can largely be explained by the increase in plasma concentrations (Fig. 1). These data suggest that blood-brain barrier Pgp/Bcrp does not (or may not) impair ketamine brain concentration. So why are the effects of Pgp/Bcrp on ketamine's PD consistent after either intraperitoneal or oral dosing, mirrored by their dual inhibitor elacridar, and consistent with oral ketamine PK, but inconsistent with ketamine's brain concentration? There are several alternative reasons for why a measurable effect of the transporters on the ketamine brain concentration at 15 minutes (HPLC-UV) and 1.25 hours (radioactivity) has not been found. First, most studies that have shown a significant effect of Pgp/Bcrp on substrate brain concentrations in vivo have measured drug brain levels 4-24 hours after drug administration to allow brain accumulation and hence maximize the difference in brain substrate concentration in the KO versus WT mice. However, because of ketamine's rapid clearance rate (estimated half-life of 25 minutes) (Supplemental Table 1), brain concentrations would not be measureable hours after dosing. Second, ketamine is a high-permeability rapidclearance drug and the magnitude of effect of Pgp and Bcrp is typically greater for substrates with poor apparent permeability coefficients (Tweedie et al., 2013), so the magnitude of transporter effect on this type of substrate would be assumed to be small. Indeed, terfenadine, another drug with high passive permeability that is a Pgp substrate, showed no difference in brain uptake clearance (measured by in situ brain perfusion) between WT and Pgp KO mice (Zhao et al., 2009). Third, it was previously shown that intravenously administered ketamine demonstrates significant regional differences in brain exposure (which are thought to be pertinent to its effect on anesthesia) during LORR in rats, and the difference minimizes as the rats regain their righting reflex (Cohen et al., 1973), which might explain its PD effect but would not be captured by measuring ketamine in total brain homogenate (as we did in this study). Hence, it is possible that the regional concentration difference of ketamine could be driving its dLORR effect, and ketamine plasma and total brain concentrations may not be a good predictor of its PK/PD relationship in the dLORR model. Fourth, we administered ketamine as the racemic mixture but it is entirely possible that the $S$ - and $R$-ketamine enantiomers (which have enantiomer-specific pharmacology) might interact differently with the transporters (beyond the scope of this work). Finally, we think the striking effect of transporter genotype on ketamine PD, but not systemic or total brain distribution, for brain-acting drugs like ketamine that have high passive permeability may, in fact, point to the potential for missing a Pgp or Bcrp effect if only the PK is measured and used to predict whether these transporters would influence the drug's central nervous system PD activity.

A population PK model was used for simultaneous analysis of intraperitoneal and oral PK data to enable estimation of fixed parameters and sources of variability. Assuming a $100 \%$ bioavailability after intraperitoneal dosing $(\mathrm{F} 2=1$; Table 1$)$, the population oral bioavailability in our study was estimated to be $17.5 \%$, which is in accordance with published reports on ketamine bioavailability in humans after oral dosing. Population analysis of intraperitoneal and oral data together identified a change in oral bioavailability in the Pgp and dKO groups. The mean population clearance for ketamine was $20.2 \mathrm{l} / \mathrm{h}$ per kilogram and the volume of distribution was $9.32 \mathrm{l} / \mathrm{kg}$, suggesting that ketamine has a very high clearance and volume of distribution, which is in accordance with previously published results (Mion and Villevieille, 2013).

Although our in vivo data clearly show that Pgp and Bcrp alter the oral PK and dLORR of ketamine, we wanted to know whether the same is observed in vitro. Previously published results show that ketamine does not have any interaction with PGP, as a substrate or inhibitor. However, our data clearly indicate, in a dual BCRP and PGP transfected MDCKII cell line, that these transporters can effectively reduce ketamine uptake in MDCKII cells at a low ketamine concentration. We think both the selection of ketamine concentration as well as the time of the in vitro assay will have a significant effect on the outcome. In addition, our in vitro data suggest that effects of these transporters will be significant at ketamine exposure lower than $10 \mu \mathrm{M}$ or $2.4 \mu \mathrm{g} / \mathrm{ml}$. A higher clinical ketamine concentration is required for the anesthetic effects of ketamine, whereas lower concentrations are useful for antidepressant effects and are also often achieved in ketamine abusers. Thus, our data warrant a possible drug-drug or food-drug interaction for antidepressant use of ketamine and in ketamine abusers. 
The finding that ketamine appears to be a dual Pgp/Bcrp substrate has a number of important clinical implications. Ketamine is an anesthetic that is widely used in animal studies and in human pediatric settings where short-acting rapid-onset anesthesia is needed. Importantly, it was recently recognized that ketamine can elicit rapid-onset long-acting antidepressant activity. However, ketamine is also a significant drug of abuse, particularly in Asian countries. Notably, reports have also appeared in the past few years and show that chronic ketamine abusers develop serious lower urinary tract symptoms, voiding problems, ulcerative cystitis, and significant damage to the urinary tract (Chu et al., 2008), although there may be individual differences in vulnerability. Both Pgp and Bcrp are expressed in the human kidney at the proximal tubule epithelium where they move substrates into the urine, and both transporters may be expressed in the bladder urothelium (Bexten et al., 2015) at the apical epithelium. Given our findings that ketamine is an apparent Pgp/Bcrp substrate, it is tempting to speculate that BCRP genetic variation, particularly the BCRP Q141K allele that leads to a loss of functional BCRP protein and has a high frequency in some populations (Zamber et al., 2003), may influence susceptibility to ketamine-induced urinary tract damage. Equally important is the potential for coadministered Pgp/Bcrp substrates or inhibitors to affect both ketamine bioavailability and urinary tract damage. Indeed, our studies with the dual Pgp/Bcrp inhibitor elacridar demonstrated that pharmacological inhibition of the transporters can affect ketamine PD. Furthermore, cyclosporine, a known Pgp/Bcrp as well as cytochrome P450 inhibitor, has similarly been shown to affect ketamine dLORR in mice (Sato et al., 2007). Many therapeutically used drugs and chemicals present in food are inhibitors of Bcrp and Pgp (Kusuhara et al., 2012). Thus, our results suggest a potential interaction of ketamine with these Bcrp-Pgp inhibitors. Moreover, the proposed use of ketamine as an antidepressant may require dosing with other antidepressant drugs, some of which are known inhibitors of these transporters (Weiss et al., 2003).

We conclude that Bcrp and Pgp can affect the PK and PD of ketamine in mice. Using a pharmacological inhibitor of Bcrp and Pgp function, our data clearly demonstrate the drug-drug interaction potential of ketamine with inhibitors of Bcrp and Pgp. Further studies should be carried out to understand the impact of these transporters on ketamine PK, PD, and drug-drug interaction potential in humans and whether persons with polymorphic Bcrp taking Pgp inhibitors have an increased risk of ketamine-mediated toxicity.

\section{Acknowledgments}

We gratefully acknowledge the technical support of St. Jude Children's Research Hospital, including the Animal Resource Center, the Hartwell Center for DNA sequencing, and the Computational Biology and Bioinformatics Core. We acknowledge Dr. Clinton Stewart's laboratory and St. Jude Children's Research Hospital Department of Pharmaceutical Sciences laboratory members for assistance with HPLC analysis of ketamine.

\section{Authorship Contributions}

Participated in research design: Ganguly, Schuetz.

Conducted experiments: Ganguly.

Contributed new reagents or analytic tools: Ganguly.

Performed data analysis: Ganguly, Panetta, Roberts, Schuetz.

Wrote or contributed to the writing of the manuscript: Ganguly, Panetta, Roberts, Schuetz.

\section{References}

Barrett TW and Schriger DL (2016) Move over morphine: is ketamine an effective and safe alternative for treating acute pain?: answers to the September 2015 Journal Club. Ann Emerg Med 67:289-294.

Bexten M, Oswald S, Grube M, Jia J, Graf T, Zimmermann U, Rodewald K, Zolk O, Schwantes U, Siegmund W, et al. (2015) Expression of drug transporters and drug metabolizing enzymes in the bladder urothelium in man and affinity of the bladder spasmolytic trospium chloride to transporters likely involved in its pharmacokinetics. Mol Pharm 12:171-178.
Bolze S and Boulieu R (1998) HPLC determination of ketamine, norketamine, and dehydronorketamine in plasma with a high-purity reversed-phase sorbent. Clin Chem 44: $560-564$

Can A, Zanos P, Moaddel R, Kang HJ, Dossou KS, Wainer IW, Cheer JF, Frost DO, Huang XP, and Gould TD (2016) Effects of ketamine and ketamine metabolites on evoked striatal dopamine release, dopamine receptors, and monoamine transporters. J Pharmacol Exp Ther 359:159-170.

Chu PS, Ma WK, Wong SC, Chu RW, Cheng CH, Wong S, Tse JM, Lau FL, Yiu MK, and Man CW (2008) The destruction of the lower urinary tract by ketamine abuse: a new syndrome? BJU Int 102:1616-1622.

Cohen ML, Chan SL, Way WL, and Trevor AJ (1973) Distribution in the brain and metabolism of ketamine in the rat after intravenous administration. Anesthesiology 39:370-376.

Durmus S, Sparidans RW, Wagenaar E, Beijnen JH, and Schinkel AH (2012) Oral availability and brain penetration of the B-RAFV600E inhibitor vemurafenib can be enhanced by the P-glycoprotein (ABCB1) and breast cancer resistance protein (ABCG2) inhibitor elacridar. Mol Pharm 9:3236-3245.

Evans AM, DeHaven CD, Barrett T, Mitchell M, and Milgram E (2009) Integrated, nontargeted ultrahigh performance liquid chromatography/electrospray ionization tandem mass spectrometry platform for the identification and relative quantification of the small-molecule complement of biological systems. Anal Chem 81:6656-6667.

Fanta S, Kinnunen M, Backman JT, and Kalso E (2015) Population pharmacokinetics of S-ketamine and norketamine in healthy volunteers after intravenous and oral dosing. Eur J Clin Pharmacol 71:441-447.

Hendrikx JJ, Lagas JS, Wagenaar E, Rosing H, Schellens JH, Beijnen JH, and Schinkel AH (2014) Oral co-administration of elacridar and ritonavir enhances plasma levels of oral paclitaxel and docetaxel without affecting relative brain accumulation. $\mathrm{Br} J$ Cancer 110: 2669-2676.

Hijazi Y and Boulieu R (2002) Contribution of CYP3A4, CYP2B6, and CYP2C9 isoforms to $\mathrm{N}$-demethylation of ketamine in human liver microsomes. Drug Metab Dispos 30:853-858.

Jaki T and Wolfsegger MJ (2011) Estimation of pharmacokinetic parameters with the R package PK. Pharm Stat 10:284-288

Keiser M, Hasan M, and Oswald S (2018) Affinity of ketamine to clinically relevant transporters. Mol Pharm 15:326-331.

Kuhn E and Lavielle M (2005) Maximum likelihood estimation in nonlinear mixed effects models. Comput Stat Data Anal 49:1020-1038.

Kusuhara H, Furuie H, Inano A, Sunagawa A, Yamada S, Wu C, Fukizawa S, Morimoto N, Ieiri I, Morishita M, et al. (2012) Pharmacokinetic interaction study of sulphasalazine in healthy subjects and the impact of curcumin as an in vivo inhibitor of BCRP. $\mathrm{Br} J$ Pharmacol 166: 1793-1803.

Lagas JS, van Waterschoot RA, van Tilburg VA, Hillebrand MJ, Lankheet N, Rosing H, Beijnen $\mathrm{JH}$, and Schinkel AH (2009) Brain accumulation of dasatinib is restricted by P-glycoprotein (ABCB1) and breast cancer resistance protein $(\mathrm{ABCG} 2)$ and can be enhanced by elacridar treatment. Clin Cancer Res 15:2344-2351.

Lilius TO, Jokinen V, Neuvonen MS, Niemi M, Kalso EA, and Rauhala PV (2015) Ketamine coadministration attenuates morphine tolerance and leads to increased brain concentrations of both drugs in the rat. Br J Pharmacol 172:2799-2813.

Machado-Vieira R, Salvadore G, Diazgranados N, and Zarate CA, Jr (2009) Ketamine and the next generation of antidepressants with a rapid onset of action. Pharmacol Ther 123: 143-150.

Merino G, van Herwaarden AE, Wagenaar E, Jonker JW, and Schinkel AH (2005) Sex-dependent expression and activity of the ATP-binding cassette transporter breast cancer resistance protein (BCRP/ABCG2) in liver. Mol Pharmacol 67:1765-1771.

Mion G and Villevieille T (2013) Ketamine pharmacology: an update (pharmacodynamics and molecular aspects, recent findings). CNS Neurosci Ther 19:370-380.

Moore KA, Sklerov J, Levine B, and Jacobs AJ (2001) Urine concentrations of ketamine and norketamine following illegal consumption. J Anal Toxicol 25:583-588.

Niesters M, Martini C, and Dahan A (2014) Ketamine for chronic pain: risks and benefits. Br J Clin Pharmacol 77:357-367.

Peltoniemi MA, Saari TI, Hagelberg NM, Laine K, Neuvonen PJ, and Olkkola KT (2012) St John's wort greatly decreases the plasma concentrations of oral S-ketamine. Fundam Clin Pharmacol 26:743-750.

Poller B, Wagenaar E, Tang SC, and Schinkel AH (2011) Double-transduced MDCKII cells to study human P-glycoprotein (ABCB1) and breast cancer resistance protein (ABCG2) interplay in drug transport across the blood-brain barrier. Mol Pharm 8:571-582.

Sane R, Agarwal S, and Elmquist WF (2012) Brain distribution and bioavailability of elacridar after different routes of administration in the mouse. Drug Metab Dispos 40: $1612-1619$

Sato Y, Kobayashi E, Hakamata Y, Kobahashi M, Wainai T, Murayama T, Mishina M, and Seo N (2004) Chronopharmacological studies of ketamine in normal and NMDA epsilon1 receptor knockout mice. Br J Anaesth 92:859-864.

Sato Y, Onaka T, Kobayashi E, and Seo N (2007) The differential effect of cyclosporine on hypnotic response and pain reaction in mice. Anesth Analg 105:1489-1493.

Shahani R, Streutker C, Dickson B, and Stewart RJ (2007) Ketamine-associated ulcerative cystitis: a new clinical entity. Urology 69:810-812.

Sherwin CM, Stockmann C, Grimsrud K, Herd DW, Anderson BJ, and Spigarelli MG (2015) Development of an optimal sampling schedule for children receiving ketamine for short-term procedural sedation and analgesia. Paediatr Anaesth 25:211-216.

Sleigh J, Harvey M, Voss L, and Denny B (2014) Ketamine - more mechanisms of action than jus NMDA blockade. Trends Anaesth Crit Care 4:76-81.

Tournier N, Chevillard L, Megarbane B, Pirnay S, Scherrmann JM, and Decleves X (2010) Interaction of drugs of abuse and maintenance treatments with human P-glycoprotein (ABCB1) and breast cancer resistance protein (ABCG2). Int J Neuropsychopharmacol 13 905-915.

Tweedie D, Polli JW, Berglund EG, Huang SM, Zhang L, Poirier A, Chu X, and Feng B (2013) Transporter studies in drug development: experience to date and follow-up on decision trees from the International Transporter Consortium. Clin Pharmacol Ther 94: 113-125.

Varma MV, Ashokraj Y, Dey CS, and Panchagnula R (2003) P-glycoprotein inhibitors and their screening: a perspective from bioavailability enhancement. Pharmacol Res 48: 347-359. 
Weiss J, Dormann SM, Martin-Facklam M, Kerpen CJ, Ketabi-Kiyanvash N, and Haefeli WE (2003) Inhibition of P-glycoprotein by newer antidepressants. J Pharmacol Exp Ther 305:197-204.

Winters WD, Hance AJ, Cadd GC, and Lakin ML (1986) Seasonal and sex influences on ketamine-induced analgesia and catalepsy in the rat; a possible role for melatonin. Neuropharmacology 25:1095-1101.

Zamber CP, Lamba JK, Yasuda K, Farnum J, Thummel K, Schuetz JD, and Schuetz EG (2003) Natural allelic variants of breast cancer resistance protein (BCRP) and their relationship to BCRP expression in human intestine. Pharmacogenetics 13:19-28.

Zanos P, Moaddel R, Morris PJ, Georgiou P, Fischell J, Elmer GI, Alkondon M, Yuan P, Pribut HJ, Singh NS, et al. (2016) NMDAR inhibition-independent antidepressant actions of ketamine metabolites. Nature 533:481-486.
Zhao R, Kalvass JC, Yanni SB, Bridges AS, and Pollack GM (2009) Fexofenadine brain exposure and the influence of blood-brain barrier P-glycoprotein after fexofenadine and terfenadine administration. Drug Metab Dispos 37:529-535.

Address correspondence to: Dr. Erin G. Schuetz, Department of Pharmaceutical Sciences, St. Jude Children's Research Hospital, 262 Danny Thomas Place, Room 15303 Chili Care Center, MS 313, Memphis, TN 38105. E-mail: erin.schuetz@stjude.org 